\title{
Virtual Reality-based Intelligent Table Lamp
}

\author{
Yingjie Fu, Dongjie Li ${ }^{*}$, Jian Song, Yujia Sheng \\ Institute of Electromechanical Control and Automation Technology, Harbin \\ University of Science and Technology, Harbin 150080, China \\ dongjieli2013@163.com
}

\begin{abstract}
With the development of modern technology, the intelligent household appliances are more and more popular among people. However, now the lamps on the market are mostly ordinary lamps, and they can not meet the requirements of users. Because of that situation, in this paper, an intelligent lamp based on virtual reality and face/book and voice recognition is designed. Firstly, the master control circuit and peripheral functional circuit of the intelligent lamp are designed with the key of ATMEGA2560 micro dips, and the body of the lamp is designed. At the same time, the virtual environment carrying virtual three-dimensional model is developed using Java (based on cell phone) and $C++$ (based on personal computer). The Open CV algorithms are used to identify images of objects that the intelligent lamp uploads. Users can interact with intelligent lamp between peripheral functional circuit and virtual environment. There are many characteristics of this intelligent lamp, such as, variety of control mode, high intelligent, stable performance, simple operation, high degree of user experience and so on. Users can modify the PC code and add related module at the reserved ports of the lamp to achieve more functions.
\end{abstract}

Keywords: intelligent lamp, virtual reality, face detection, ATMEGA chip

\section{Introduction}

With the development of the electronic technology, the requirement of electronics products like lamp has increased highly [1]. Currently, there are many kinds of lamp on the market, but most of them focus on simple functions such as the exterior design of lamp or lighting brightness adjustment $[2,3]$. The model of traditional lamp is single, and only has a simple user experience. Their functions are similar [4-7]. But these improvements did not fundamentally solve the problem of single model, low degree of intelligence, simple function and low degree of user experience $[8,9]$. In abroad, the studies of desk lamp are more mature than domestic, and the lamp is focused on the user experience [10]. The wireless charging technology has been successfully integrated into the lamp, and simple user interaction has been achieved. The features of the product are rich [11, 12]. However, the interactive intelligent lamp with positioning on the face has not yet appeared [13-15]. So, it is necessary to develop such an intelligent lamp, to meet the needs of the market.

According to the above situation, the intelligent lamp is designed with the function of virtual model interaction and face/book, voice recognition. Then the control circuit and peripheral function circuit are built based on ATMEGA 2560 chip. The peripheral circuits include: the voice recognition circuit (used to implement voice operation of desk lamp), the light detecting circuit (used to test the strength of the light in the surrounding), the image detecting circuit (used to collect environmental image and identify the objectives such as books and face), the storage circuit (used to store instructions and initialization information), the communication circuit (used to implement the remote communication function), the buck 
circuit (used to handle voltage to fulfill the requirements of desk lamp), the touch control circuit, the actuator (used to implement the mechanical action of desk lamp) and the main control circuit equipped with lower computer program, it can deal with the signal of voice recognition, light detection circuit, steering gear, light detection circuit and can communicate with the Personal Computer(PC) software and data interaction.

\section{Design of Lamp Body}

The lamp mainly consists of the body, hardware part and software part. The body comprises the substrate, the light pole, the special lamp shade, the special steering gear shaft in vertical and horizontal and its artifacts. The structure of lamp body is shown in Figure 1. The substrate is circular, and has built-in core control module, light detection module, brightness control module, storage module, wireless communication module, buck module and touch switch. The light pole is two hollow cylindrical plastic trestles, and the two trestles are connected with cardan joint, it can be positioned between 30 degrees to 180 degrees. The pole and the substrate are connected by bolts. The top of the pole mounted vertically with two high torque metal steering gear control the special shaft driven lamp to achieve 300-degree horizontal rotation and 180 degrees vertical rotation. The special shaft above the case of loading the steering gear, and it is connected with the horizontal and vertical steering gear respectively. The special lamp shade is conical; connect with the special shaft through the bolt. The camera is equipped outside of the shade. The lamp holder is on the bottom of the lamp shade. There is a bulb be equipped in special lamp shade for lighting.

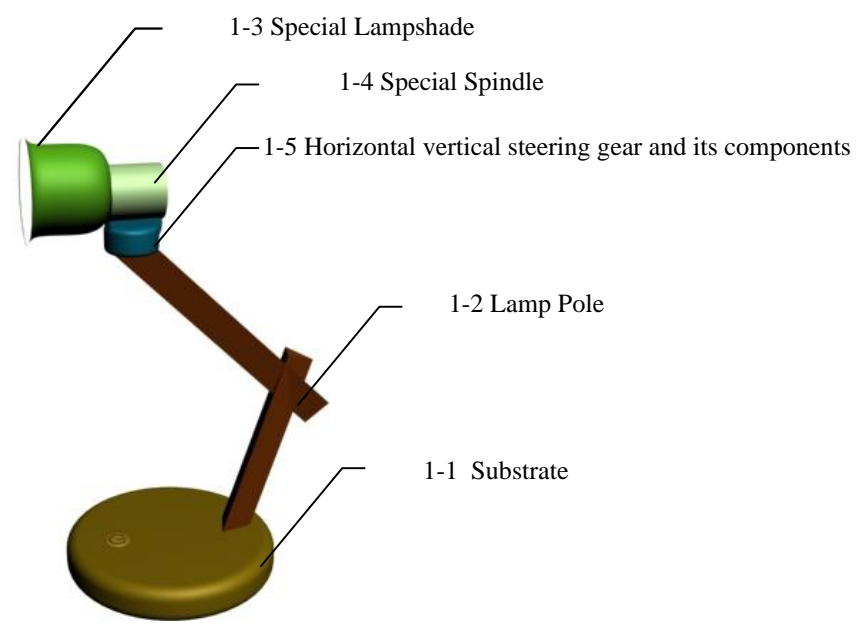

Figure 1 The Structure of Lamp Body

\section{Realization of the Lamp Function}

The intelligent lamp mainly designed based on virtual reality has the following five functions: voice recognition function, light adjustment function, face/books identify and locate function and wireless control functions. In addition, the user can add other hardware circuits in extension port of the hardware reserved, then, the appendant open source code of the product and the PC program can be modified to support the newly added hardware. The structure of intelligent lamp system functions is shown in Figure 2. 


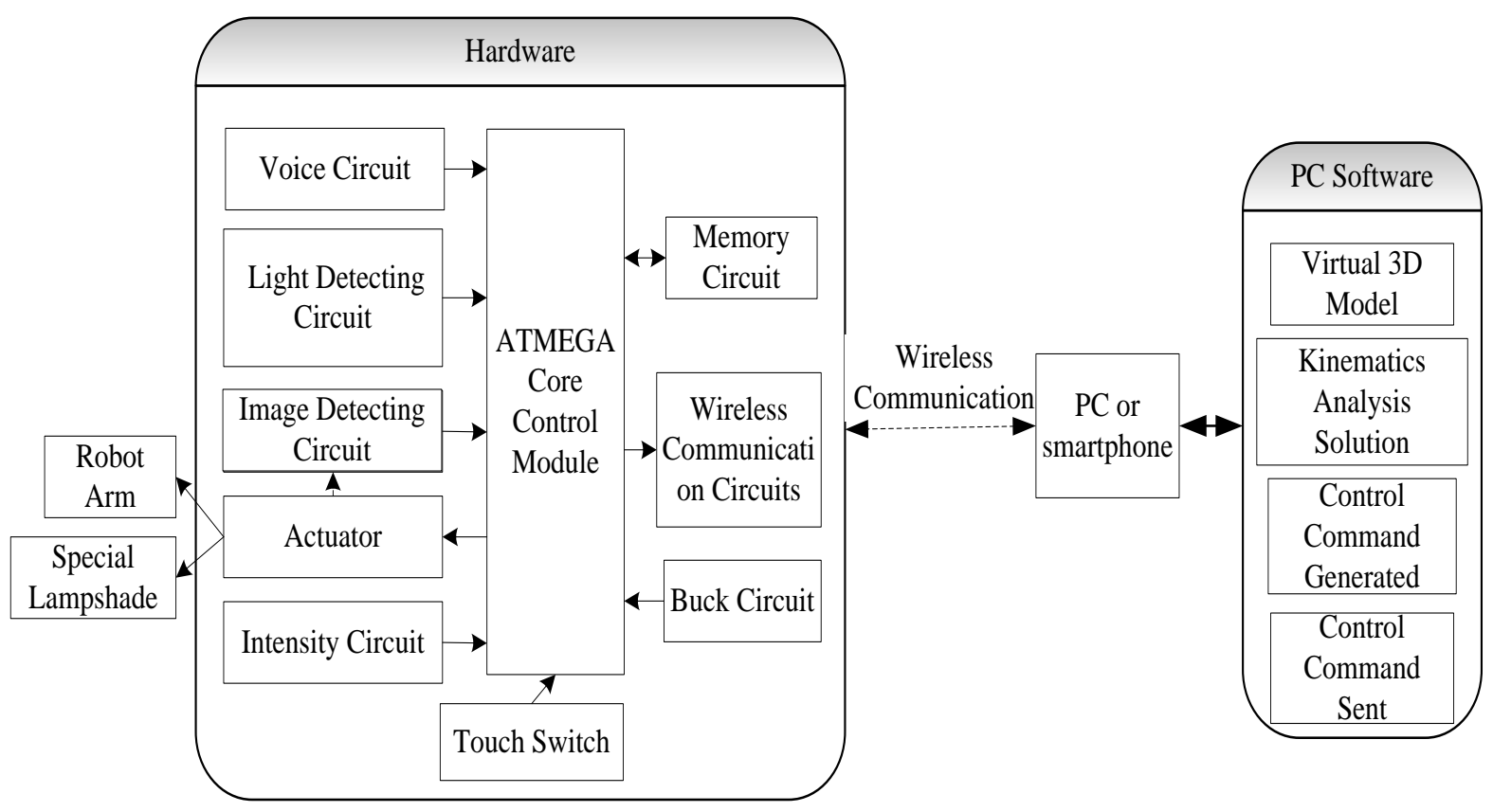

Figure 2 The Structure of Intelligent Lamp System Functions

The Atmega 2560 chip has large numb of available resources. It is reserved many ports in development and suit for requiring a lot of IO interface. The single chip of using the ATMEGA chip as the core has been used in intelligent lamp development. Its expansion port can be used to integrate circuits required, to achieve specific functions.

\subsection{Voice Recognition Module}

Voice recognition circuit consists of the existing LD3320 circuit; this circuit can identify non-specific voice, then, collect and analysis the voice signal through adding the microphones and speakers, and enter the microcontroller core control circuit. The microcontroller core control circuit controls the actuator to make lamps do the appropriate action after comparison and processing.

\subsection{Light Adjustment Module}

The light detection circuit composed of BH1705 chip, peripheral resistance and capacitance. The circuit is controlled by I2C bus. It has high detection accuracy. The intelligent lamp collects the intensity of the light surroundings through the light detection circuit, then through controlling the size of current to adjust the brightness of intelligent lamp automatically after analog-to-digital conversion and analysis of the core control circuit.

\subsection{The Identification and Location of Facebook}

Image detection circuit composed of OV7670 CMOS VGA image sensor and its peripheral circuit. The image sensor with resolution of $640 * 480$ can realize automatic detection of $50 / 60 \mathrm{~Hz}$, and the maximum frame rate of VGA images can be 30 frames/sec, which meets the needs of intelligent lamp. The connections of chip pin is shown in Figure 3. The camera captures surrounding images in fixed time, then, the image is stored in the memory circuit chip. The file that trained using OpenCV machine 
learn algorithms can automatically identify and locate the face or book in images and can calculate the book/face position using a specific algorithm, and the displacement of the steering gear. The actuator is composed of specific placement of metal servos and linkage. It can drive the corresponding mechanical arm movement according to the collected information to change the direction of the lamp shade and the camera. So the function of identifying and positioning face and books of the intelligent lamp is realized.

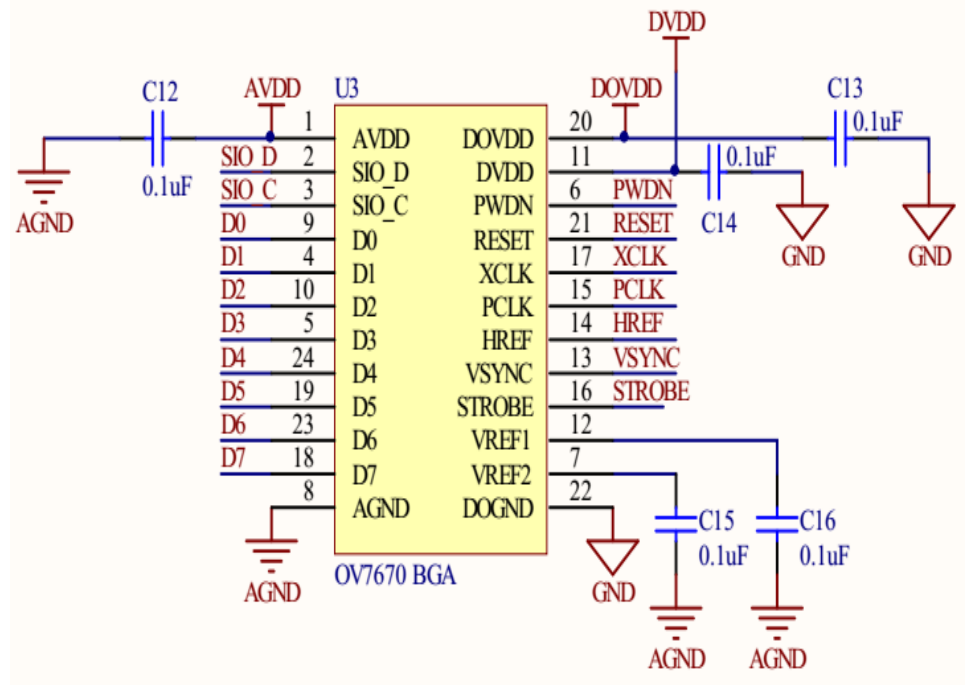

Figure 3 Connections Method of OV7670 Chip Pin

\subsection{Wireless Control Module}

The lamp also has the wireless communication function; the communication circuit adopts ZigXbee as communication carrier, and connects with a serial port. The ZigXbee can be arbitrary set up Potter road performance.

The core control circuit of signal chip equipped with lower computer program can handle voice recognition, light detection circuit, steering gear, and the signal of light detection circuit. The lower computer program can receive and process the data of wireless communication circuit, and make corresponding movement. It also can send the current encoded location information of the lamp through wireless communication circuit. The lower machine program adopts the fuzzy PID method to control the steering gear. This method can make the operation of the steering gear stable, the action gently, small overshoot and short time of movement to the destination. The lower computer program can send images that captured under special control of the camera through wireless communication mode.

\subsection{Establishment of the Virtual Lamp Model}

PC software mainly consists of a virtual three-dimensional lamp model, kinematics algorithms, image processing algorithms, control command generating/transmitting algorithm and so on. The virtual three-dimensional lamp is developed using $\mathrm{C}++$ language in 3DsMax aid, and combining with OpenGL modeling language. The virtual three-dimensional model has been established is corresponding with the actual lamp at fixed zoom ratio. The three-dimensional model of lamp is got after 3DsMax designed 
and the $\mathrm{C}++$ file of three-dimensional model obtained by file transforming. Then it is imported in VS2008. The parts of lamp are been correspond to each parameter with OpenGL programming. Through design algorithm, the two-dimensional information is mapped to the three-dimensional space, so that changes of the mouse position transfer into the posture changes of different parts of the lamp. In the operation, the virtual lamp model can use a mouse or a finger to change the relative position between the different structures, and this position information are sent to the actual lamp through wireless communication module, then, the control module of the actual lamp analyzes the information and outputs the appropriate motion.

The PC software developed can receive and analyze the wireless information, and automatic access information in the image. Then the face recognition file and the trained book recognition file with Open $\mathrm{CV}$ algorithms are used to identify images of objects that the intelligent lamp upload, extract the location information of the object automatically, and calculate the servo operation mode. It also can make inverse kinematics analysis based on the relative position and orientation of a virtual model to get the actual lamp operating parameters. These parameters are shown in the interactive interface for users to reference. As shown in Figure 4 and Figure 5, the intelligent lamp human-computer interaction diagrams based on PC and smart phone are shown respectively.

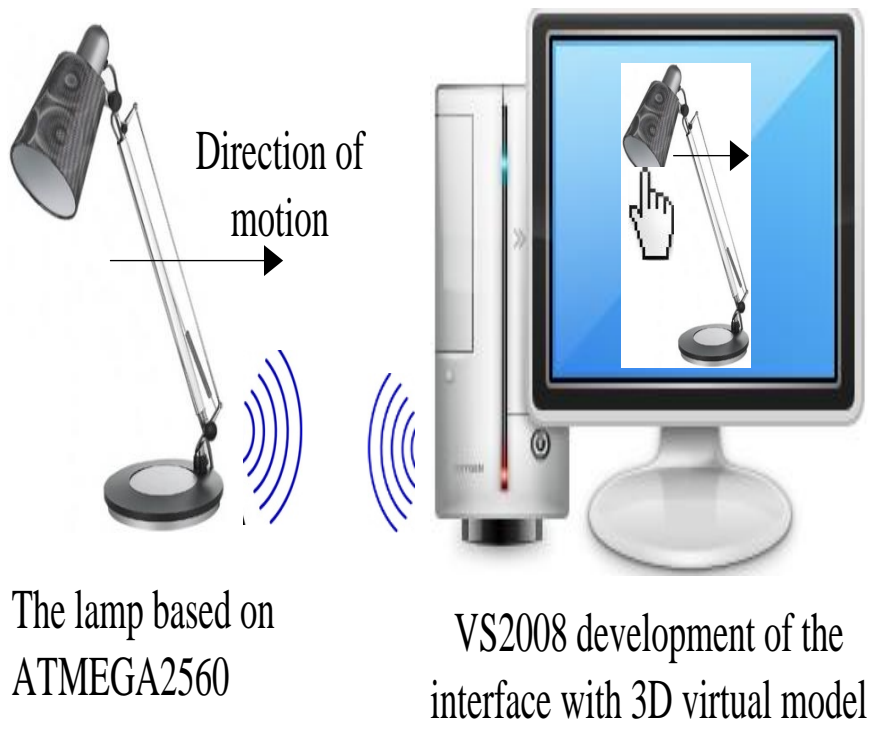

Figure 4 The Diagrams of Lamp Human-Computer Interaction with PC

\section{The Working Process of the Intelligent Lamp System}

After the intelligent lamp powered, the initialized position of lamp, the light intensity parameters and the lamp holder are in a predetermined position under the control of signal chip. After the control circuit detects the surrounding light conditions, the lighting current is automatically adjusted, and the predetermined brightness of intelligent lamp is reached. The lamp can search wireless signals and sound signals under the control of control circuit, and switch to the virtual model control or voice control mode according to the presence of wireless signals and sound signals. If the 


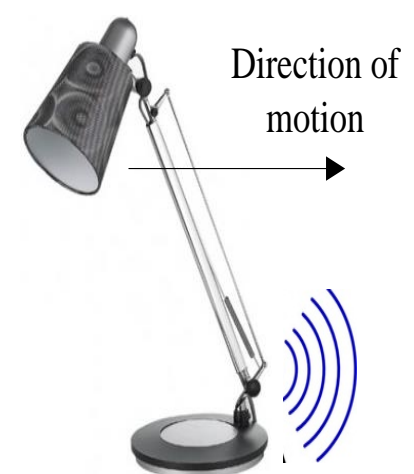

The lamp based on ATMEGA2560

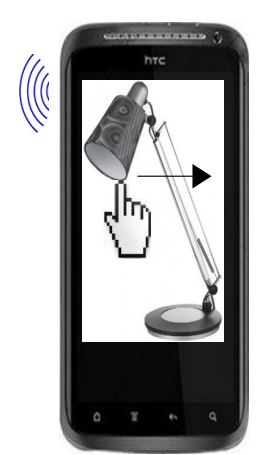

JAVA development of virtual operation interface based on Android

\section{Figure 5 The Diagrams of Lamp Human-Computer Interaction with Smart Phone}

lamp enters the virtual model control mode, the information that obtained from the wireless communication is analyzed by control circuit, and the control command is generated, then, the actuator makes appropriate motion. If the lamp enters the voice control mode, the control circuit detects the sound signal identified, and the control command is generated, then, the actuator makes appropriate motion. During operation of the intelligent lamp, the camera shoots surrounding at predetermined time intervals according to the voice command, and uploads the images. These images are recognized with Open $\mathrm{CV}$ algorithms and decisionmade by the Personal Computer (PC). Then, the book or face is tracked automatically according to the decision command.

The recognition of face or book is achieved by processing the image that image sensor obtained. The camera is located in the top of the special lamp shade. The sensor obtains surrounding images at a predetermined time when it is working. The images are demarcated after the target in images is recognized by the recognition file of face or book. Then the center position of face or book is calculated and compared with the center position of image to obtain the deviation between both. At last, it is converted to the offset of the steering gear after calculated. The workflow is shown in Figure 6.
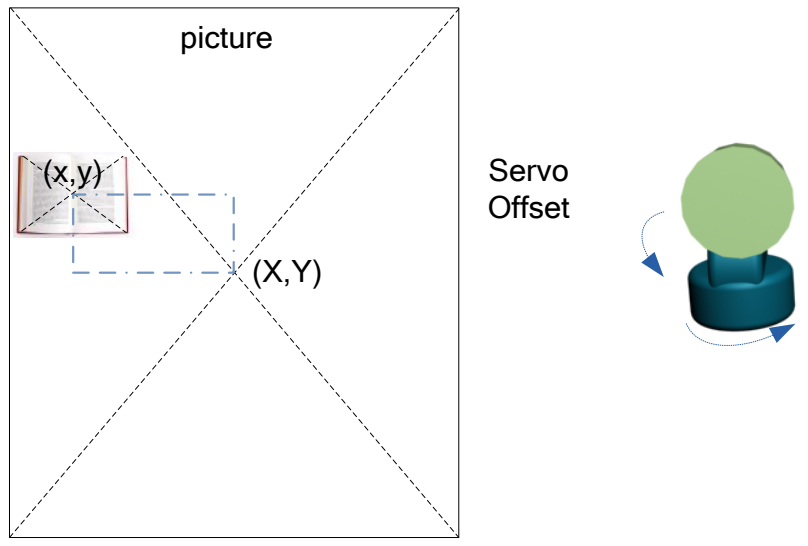

Figure 6 The Workflow of the Recognition of Face or Book 


\section{Control Modes of the Intelligent Lamp}

The intelligent lamp designed can be controlled with three modes: the voice control mode, the virtual model control mode and the manual control mode. The voice control mode gathers the voice signals though the voice recognition circuit. The signals are inputted core control circuits of signal chip after recognition. Through analysis and comparison, the actuator is controlled by the core control circuit of signal chip to make corresponding action. The virtual model control mode is achieved through the $\mathrm{PC} /$ smart phone operation, the PC/smart phone equipped with a program that establishes the virtual three-dimensional model which is corresponding with real lamp in proportion. With controlling the virtual model and analyzing the kinematics algorithms, the commands are generated by the control command generating module, and are sent by the control command transmitting module. After the date is received by communication circuit, the core control circuits of signal chip analysis that the action of actual lamp consistent with the virtual action. Manual control is relatively simple, only to adjust the brightness of the light by touching the control circuit, and turn on/off through an external switch.

\section{Conclusions}

In this paper, an intelligent lamp with voice and virtual model interacting was designed. The body structure and the function realization of the intelligent lamp were designed in detail. This lamp can automatically find book and face according the corresponding instruction, automatically adjust the lighting strength and the light focus. It can make the appropriate action to achieve more interaction with humans. This product makes the lamp really intelligent, convenient and feature-rich. The experience of user is greatly increased. It can change the traditional habit of using lamp and effectively solve problems that current market faced single mode, low degree of intelligence and low degree of user experience.

The intelligent lamp, which based on virtual reality interactive, can be operated by the virtual model software, voice, manual operation mode. This intelligent lamp is not only a lamp, but also an intelligent robot. Buyers can modify the codes and add related module at the reserved ports of the lamp to achieve more functions. This lamp also can be used as a tool of learning motor control, image recognition, speech development, signal chip development or robot practical to achieve more value.

\section{Acknowledgements}

This work was supported by the Undergraduate Training Programs for Innovation and Entrepreneurship of Harbin University of Science and Technology.

\section{References}

[1] X . Kai, X. Benjie and C. Xiong, "An intelligent dimming power system for LED desk amp", Proceedings of the 2012 International Conference on Industrial Control and Electronics Engineering, Xi'an, China, August (2012).

[2] X. Wilsunand and J. Novaks, "Power electronic technology", Machinery industry press, (2000).

[3] Y. Huiwei, "BISS0001 based intelligent lamp design", Journal of electronic applications, vol. 27, no. 4, (2010).

[4] Yuanjie. D, "Single chip microcomputer principle and application [M]", Mechanical industry publishing club, (2005).

[5] Z. Jiwen, "Sensor and application circuit design", Science press, (2011). 
[6] L. Xinhua, H. Pan and L. Fangmin, "A street lamp clustered-control system based on wireless sensor and actuator networks", Proceedings of the World Congress on Intelligent Control and Automation (WCICA), Beijing, China, July (2012).

[7] Z. Zhimin, "The design and application of driving circuits for LED", The press of People Posts and Telecommunications, (2009).

[8] X. Yibo and C. Wenqing, "Foundation and application of switch power source", The press of Xidian University, (2009).

[9] M. Tata, "Method for detecting the head lamp switch failure or wiring harness failure and controlling the exterior and interior lights using intelligent light sensor", SAE 2011 World Congress and Exhibition, Detroit, MI, United states, (2011) April.

[10] S. Cheng, "Rich homemade improvised dimmer lamp", The home court electronics, (1997).

[11] L. Kiyak, O. Bulent and V. Topuz, "Design and comparative analysis of high power led with fluorescent lamp in laboratory lighting systems", Energy Education Science and Technology Part A: Energy Science and Research, vol. 2, no. 29, (2012).

[12] C. Cianci, M. Christopher and J. Nembrini, et.al., "Assembly of configurations in a networked robotic system: A case study on a reconfigurable interactive table lamp", 2008 IEEE Swarm Intelligence Symposium, St. Louis, MO, United states, (2008) September.

[13] T. Dong-Sheng, H. Zheng, X. L. Yao and W. Sun et.al., "Three-dimensional visualization calculation and research on dynamic simulation of landslide based on Open GL", Journal of Railway Engineering Society, vol. 8, no. 30, (2013).

[14] O. Gustavo, L. Charlier and Baudouin, "Formalization of C Language Interfaces", Electronic Notes in Theoretical Computer Science, vol. 4, no. 229, (2009).

[15] Gunes, Hatice, Piccardi and Massimo, "Automatic visual recognition of face and body action units", Proceedings-3rd International Conference on Information Technology and Applications, Sydney, Australia, (2005) July. 